\title{
ESTUDO CLfNICO DE 68 CASOS DE EPILEPSIA OCCIPITAL
}

\author{
Luís MARQues-Assis * \\ José Antonio Livramento *: \\ Michel CURY **
}

As epilepsias com disritmia occipital ao eletrencefalograma são particularmente freqüentes na infância ${ }^{2,3,8}$. Embora exista uma tendência à normalização do traçado das crianças com epilepsia occipital ${ }^{3}$, não é raro que se encontre, no paciente epiléptico adulto, disritmia com essa localização.

No presente trabalho propomo-nos a estudar, do ponto de vista clínico, 68 casos de epilepsia, adultos na sua maioria, com foco occipital.

\section{MATERIAL, METODO E RESULTADOS}

Foram estudados 68 pacientes epilépticos matriculados no Ambulatório de Neurologia do Hospital das Clinicas de São Paulo. Désse total 38 eram do sexo masculino e 30 do sexo feminino. A idade estava compreendida entre 10 e 39 anos. O tempo de doença variou de uma semana a 15 anos. Em nenhum caso foi verificada a existência de sinais neurológicos focais ou de hipertensão intracraniana. Quanto a manifestçōes clinicas, 65 casos apresentavam crises convulsivas (em 50 casos as convulsões eram secundárias a descargas focais e, em 15, eram crises grande mal puras), crises motoras focais ocorreram em 11 casos, sensitivo-gensoriais em 30 , aura psiquica em 5, aura visceral em 14 e crises psicomotoras em 3 casos; em apenas 5 casos foi referida aura visual.

Em 67 pacientes foi feito exame do liquido cefalorraqueano, que foi anormal em 4 casos, nos quais foi constatado discreto aumento de células. $O$ eletrencefalograma, teito em todos os casos, mostrou anormalidades occipitais paroxisticas em 40 e contiuas em 27 casos; em um caso havia assimetria entre as áreas occipitais. A disritmia paroxística era direita em 10 casos, esquerda em 7 e bilateral em 23 casos; a disritmia contínua era direita em 7 casos, esquerda em 10 e bilateral em 12 casos (Quadro 1).

No tratamento foram utilizados, isoladamente ou em associaçăo, barbitúricos, hidantoinatos, primidona e trimetodiona. O estudo foi feito em relação ao tempo de doença, à freqüência das crises e, em 40 casos, em relação à evolução da doença, sendo comparadas as epilepsias occipitais com as epilepsias em geral ${ }^{10},{ }^{11}$. Os resultados podem ser apreciados nos quadros 2 a 6.

Trabalho realizado na Clínica Neurológica (Prof. Horácio M. Canelas) do Departamento de Neuropsiquiatria da F.M.U.S.P., apresentado no IV Congresso Brasileiro de Neurologia e II Congresso Brasileiro de Eletrencefalografia e Neurotisio logia Clinica (Porto Alegre, 5 a 9 de julho de 1970): * Médico assistente; ** Médico estagiário. 
Disritmia Paroxistica Continui occipital

\begin{tabular}{lrr}
\hline Direita & 10 & 7 \\
Esquerda & 7 & 10 \\
Bilateral & 23 & 10 \\
Assimetria & 1 & 0 \\
Total & 41 & 27 \\
\hline
\end{tabular}

Quadro 1 - Resultados do eletrencefalograma nos 68 casos de epilepsia occipital estudados.

\begin{tabular}{crrrr}
$\begin{array}{c}\text { Idade } \\
\text { de inicio } \\
\text { (anos) }\end{array}$ & $\begin{array}{c}\text { Epilepsias } \\
\text { occipitais }\end{array}$ & \multicolumn{2}{c}{$\begin{array}{c}\text { Epilepsias } \\
\text { em geral }\end{array}$} \\
\hline $0-9$ & 19 & $28 \%$ & 316 & $26 \%$ \\
$10-19$ & 39 & $57 \%$ & 578 & $47 \%$ \\
20 ou mais & 10 & $15 \%$ & 323 & $27 \%$ \\
Total & 68 & $100 \%$ & 1217 & $100 \%$ \\
\hline
\end{tabular}

Quadro 2 - Estudo comparativo entre as epilepsias occipitais (68 casos) e as epilepsias em geral em relação à idade de inicio da doença.

\begin{tabular}{|c|c|c|c|c|c|c|}
\hline \multirow{2}{*}{$\begin{array}{c}\begin{array}{c}\text { Tempo de doença } \\
\text { (anos) }\end{array} \\
\text { até } 1\end{array}$} & \multicolumn{4}{|c|}{ EEG com anormalidade } & \multicolumn{2}{|c|}{$\begin{array}{l}\text { Epilepsias } \\
\text { em geral }\end{array}$} \\
\hline & 21 & $\mathbf{5 0 \%}$ & 18 & $67 \%$ & 369 & $30 \%$ \\
\hline $1-3$ & 8 & $20 \%$ & $\mathbf{5}$ & $18 \%$ & 195 & $16 \%$ \\
\hline $3-10$ & 10 & $25 \%$ & 1 & $4 \%$ & 369 & $30 \%$ \\
\hline mais de 10 & 2 & $5 \%$ & 3 & $11 \%$ & 284 & $24 \%$ \\
\hline Total & 41 & $100 \%$ & 27 & $100 \%$ & 1217 & $100 \%$ \\
\hline
\end{tabular}

Quadro $s$ - $O$ tempo de doença nos casos com disritmia occipital (paroxistica $e$ contínua) em relacão aos casos de epilepsia em geral.

\begin{tabular}{|c|c|c|c|c|c|c|}
\hline $\begin{array}{c}\text { Freqüência } \\
\text { (dias) }\end{array}$ & $\mathrm{Pa}$ & \multicolumn{2}{|c|}{ Disritmia occipital } & inua & & $\begin{array}{l}\text { epsias } \\
\text { geral }\end{array}$ \\
\hline até $7 / 7$ & 7 & $17 \%$ & 6 & $22 \%$ & 309 & $25 \%$ \\
\hline de $8 / 8$ a $30 / 30$ & 12 & $29 \%$ & 6 & $22 \%$ & 361 & $30 \%$ \\
\hline de $31 / 31$ a $90 / 90$ & 1 & $2 \%$ & 7 & $26 \%$ & 147 & $12 \%$ \\
\hline inferion a $90 / 90$ & 10 & $25 \%$ & 3 & $11 \%$ & 187 & $15 \%$ \\
\hline formas iniciais & 11 & $27 \%$ & 5 & $19 \%$ & 213 & $18 \%$ \\
\hline Total & 41 & $100 \%$ & 27 & $100 \%$ & 1217 & $100 \%$ \\
\hline
\end{tabular}

Quadro 4 - A severidade da doença nos casos de disritmia occipital (paroxística $e$ continua) em relação aos casos de epilepsia em geral. 


\begin{tabular}{ccccc}
\hline Evolução & \multicolumn{3}{c}{$\begin{array}{c}\text { Disritmia } \\
\text { Paroxistica }\end{array}$} & $\begin{array}{c}\text { Occipital } \\
\text { Continua }\end{array}$ \\
\hline I & 2 & $9 \%$ & 3 & $16 \%$ \\
Md & 5 & $\frac{24 \%}{33 \%}$ & 0 & 0 \\
I + Md & 6 & $27 \%$ & 7 & $16 \%$ \\
Ms & 9 & $40 \%$ & 8 & $45 \%$ \\
R & & $67 \%$ & & $84 \%$ \\
Ms + R & 22 & $100 \%$ & 18 & $100 \%$ \\
Total & & & & \\
\end{tabular}

Quadro $5-A$ evolução da doença nos casos com disritmia occipital paroxística e contínua. Legenda: $I=$ inalterado, $M d=m e-$ lhora discreta, $M s=$ melhora sinsivel e $R=$ remissão.

\begin{tabular}{|c|c|c|c|c|}
\hline Evolução & \multicolumn{2}{|c|}{$\begin{array}{l}\text { Epilepsia } \\
\text { occipital }\end{array}$} & \multicolumn{2}{|c|}{$\begin{array}{l}\text { Epilepsias } \\
\text { em geral }\end{array}$} \\
\hline I & 5 & $12 \%$ & 87 & $7 \%$ \\
\hline $\mathrm{Md}$ & 5 & $12 \%$ & 55 & $4 \%$ \\
\hline $\mathbf{I}+\mathbf{M d}$ & & $24 \%$ & & $11 \%$ \\
\hline Ms & 13 & $\mathbf{3 3} \%$ & 558 & $46 \%$ \\
\hline $\mathbf{R}$ & 17 & $43 \%$ & 517 & $43 \%$ \\
\hline $\mathbf{M s}+\mathbf{R}$ & & $76 \%$ & & $89 \%$ \\
\hline Total & 40 & $100 \%$ & 1217 & $100 \%$ \\
\hline
\end{tabular}

Quadro 6 - Evolução global dos casos de epilepsia occipital em relação aos casos de epilepsia em geral.

\section{COM E N TA R I O S}

As áreas posteriores são, com muita freqüência, séde de descargas epilépticas em crianças ${ }^{2,3,8}$. As epilepsias occipitais, quando considerados pacientes adultos, são relativamente raras (Marques-Assis ${ }^{10}$, estudando 1217 pacientes epilépticos adultos em sua maioria, encontrou epilepsias focais não temporais em apenas $17 \%$ dos casos, entre os quais estavam incluidas aquelas com focos occipitais). Por outro lado, revisão da literatura demonstra que, relativamente aos aspectos clínicos e eletrencefalográficos, pouca atenção tem 
sido dada às epilepsias occipitais ${ }^{4,5,6,7,9,12}$. No presente trabalho procuramos estudar comparativamente um grupo de pacientes com epilepsia occipital e um grupo com epilepsia em geral ${ }^{10},{ }^{11}$, da mesma faixa etária (adultos em sua maioria).

A análise do quadro 2 permite verificar o início precoce da doença no grupo em estudo em relação às epilepsias em geral: em 27\% dos casos o início ocorreu após os 20 anos de idade nas epilepsias em geral enquanto que, nas epilepsias occipitais, êsse fato foi constatado em apenas $15 \%$ dos casos. No que se refere às manifestações clínicas, Penfield e Rasmussen (cit. por Penfield e Jasper ${ }^{12}$ ), observando os resultados da estimulação dos lobos occipitais de pacientes conscientes, verificaram a produção de sintomas visuais elementares; foram referidos também movimentos oculares ou de abertura e fechamento dos olhos. Cotte-Rittaud e Courton ${ }^{1}$, estudando o valor semiológico das crises adversivas, encontraram anormalidades occipitais ictais ou interictais em 12 e $7 \%$ dos casos respectivamente. Em nossos casos, afóra as manifestações convulsivas que ocorreram em 65 pacientes, em 50 dos quais representando difusão de descarga focal, em apenas 5 a sintomatologia foi de tipo visual, havendo predominio de outras manifestações sensitivo-sensoriais, viscerais e motoras focais; deve-se notar que, em apenas três casos foram referidas crises versivas, num dos quais o fóco era contra-lateral. A variedade das manifestações talvez seja explicada pela rápida difusão da descrga para áreas anteriores por ocasião da crise.

Qundo encaradas as epilepsias de modo geral, é geralmente admitido que os casos com anormalidades eletrencefalográficas contínuas sejam de maior gravidade que aquêles com anormalidade paroxistica. Levando em conta êsse fato, os pacientes com disritmia occipital foram estudados em relação ao tempo de doença, à freqüência das crises e à evolução sob tratamento medicamentoso, sendo feito estudo comparativo entre os casos com disritmia occipital paroxística e contínua em relação às epilepsias em geral ${ }^{10,11}$. No que se refere ao tempo de doença (Quadro 3) não houve diferenças significativas nas epilepsias occipitais, embora fossem observados com maior freqüência casos com mais de 5 anos de doença entre aqueles com disritmia contínua; no entanto, verifica-se que nas epilepsias occipitais houve predomínio acentuado do menor tempo de evolução em relação às epilepsias em geral. Também no que diz respeito à severidade da doença (Quadro 4) o comportamento foi semelhante nos dois grupos com epilepsia occipital; nota-se apenas maior incidência de formas leves da doença (freqüência de crises inferior a uma cada três meses ou formas iniciais) nos casos com disritmia paroxistica; quando comparadas as epilepsias occipitais com as epilepsias em geral, verifica-se que predominam nestas as formas mais severas e, naquelas, as formas moderadas da doença. O estudo da evolução (40 casos) relativamente aos grupos em estudo (Quadro 5), demonstra que melhores resultados foram obtidos em pacientes com disritmia occipital contínua, embora de maneira pouco significativa. Esse fato vem demonstrar que, sob êsse aspecto, o lobo occipital deve ser interpretado de forma diferente de outras áreas corticais, não fodendo ser a continuidade da anormalidade encarada como tradução de 
maior gravidade do caso. Por outro lado, as epilepsias de modo geral têm evolução mais favorável que as epilepsias occipitais, conforme se pode observar também pela análise do quadro 6 .

\section{RESUMO E CONCLUSOES}

Foram estudados 68 pacientes epilépticos, com disritmia occipital no eletrencefalograma. A investigação foi desenvolvida quanto ao tipo de crise, à idade de início da doença, ao tempo de doença, à freqüência das crises e à evolução com tratamento medicamentoso, relativamente às disritmias occipitais paroxísticas e contínuas. Os resultados obtidos foram comparados com as epilepsias de modo geral e analisados em indices percentuais, tendo os autores chegado às seguintes conclusões: 1) em 65 casos (94\%) havia manifestações convulsivas, sendo que em $50(73 \%)$ as convulsões eram secundárias; 2) em apenas $7 \%$ dos casos havia sintomas visuais precedendo as convulsões; 3) a doença teve início precoce nas epilepsias occipitais em relação às epilepsias em geral; 4) o tempo de doença foi menor nas epilepsias occipitais; 5) as formas mais severas da doença predominaram nas epilepsias em geral; 6) não houve diferenças marcantes, quanto à evolução, entre as epilepsias occipitais paroxísticas e continuas; 7) a evolução global das epilepsias occipitais não foi tão favorável quanto a das epilepsias em geral.

\section{S U M M A R Y}

\section{Clinical study of 68 cases of occipital epilepsy}

Sixty eight epileptic patients with occipital EEG foci were studied. The investigation was developped in cases with paroxistic and continuous electroencephalographic abnormalities, concerning the age of onset and time of disease, frequency of seizures and evolution with drug treatment. The results were compared with the epilepsies in general and analysed in percentual index. The authors draws the following conclusions: 1) 65 cases (94\%) exhibit convulsive seizures; in 50 cases $(73 \%)$ the convulsions were secondary; 2) only $7 \%$ of patients exhibit visual aura; 3 ) the disease developped earlier in the occipital epilepsy than in the epilepsy in general; 4) the duration of disease was smaller in the ocipital epilepsy; 5) the disease was less severe in the occipital epilepsy than in the epilepsy in general; 6) the evolution of the paroxistic and continuous occipital epilepsy was similar; 7) the evolution of the occipital epilepsy was worse than the epilepsy in general.

R E F E R E N I A S

1. COTTE-RITTAUD, M. R. \& COURTON, J. - Semiological value of adversive epilepsy. Epilepsia 3:151, 1962.

2. GEETS, W. \& DELANOIS, B. - Foyers occipitaux dans les épilepsies infantiles. Acta neurol. psychiat. belg. 57:318, 1957. 
3. GIBBS, E. L.; GILLEN, H. W. \& GIBBS, F. A. - Disappearance and migration of eplleptic foci in childhood. Amer. J. Dis. Child. 88:596, 1954.

4. KILOH, L. G. \& OSSELTON, J. W. - Clinical Electroencephalography. Butterworths Co., Londres, $2 .^{\circ}$ ed., 1966.

5. KUGLER, J. - La Electroencefalografia en la Clinica y en la Practica. Versão eastelhana. Ed. Alhambra, Madrid, 1969.

6. LAGET, P. \& SALBREUX, R. - Atlas d'Electroencéphalographie Infantile. Masson et Cie., Paris, 1967.

7. LENNOX, W. G. \& LENNOX, M. A. - Epilepsy and Related Disorders. LittleBrown Co., Boston, 1960.

8. LESNY, I. - EEG studies in the focal epilepsy in children. Neurol. Neuroch. Psychiat. Polska 13:791, 1963 (resumo em inglés).

9. LIVINGSTONE, S. - Convulsive Disorders in Children. Charles C. Thomas, Springfield (Illinois), 1954.

10. MARQUES-ASSIS, L. - Considerações a propósito do tratamento medicamentoso de 1217 pacientes epilépticos. Estudo em relação ao tipo de epilepsia e ao eletrencefalograma. Arq. Neuro-Psiquiat. (São Paulo) 27:312, 1969.

11. MARQUES-ASSIS, L. - Tratamento medicamentoso de 1217 pacientes epilépticos. Estudo em relação à idade de início, ao tempo de doença e à frequiência das crises. Arq. Neuro-Psiquiat. (São Paulo) 28:44, 1970.

12. PENFIELD, W. \& JASPER, H. - Epilepsy and the Functional Anatomy of the Human Brain. Little-Brown Co., Boston, 1954.

Clinica Neurológica - Faculdade de Medicina, Universidade de São Paulo Caixa Postal 3461 - Sáo Paulo, SP - Brasil. 